\title{
ON A NEW MIXED PROBLEM OF THE PARTIAL DIFFERENTIAL EQUATION OF TELEGRAPHY.
}

BY PROFESSOR ARTHUR GORDON WEBSTER.

(Read before the American Mathematical Society, September 13, 1911.)

DuRING the last twenty years the question of the propagation of electric waves in wire lines or cables has been the subject of a very great number of researches, on the one hand on account of its practical importance in connection with telephony, rapid telegraphy, wireless telegraphy, and the transmission of energy by means of alternating currents, and on the other on account of its purely mathematical interest and of the great increase in our knowledge of partial differential equations of the hyperbolic type. In all these researches the point of view has been very different in the different cases and may be roughly classified as follows. The electrical engineer, whether interested in transmission, telephony, or wireless, has been mainly concerned with phenomena depending upon simple harmonic functions of the time, that is, with standing wave phenomena after the steady state of oscillation has been established. For these the methods of trigonometric series suffice perfectly, and with them we are not here concerned. In these cases the line is of finite length, and we deal with series of functions. On the other hand the mathematician has been interested in problems connected with propagation in a line of infinite length, especially as depending on the data of the initial state of the line. For this case instead of infinite series we should use definite integrals of Fourier, but the method of Riemann has furnished a more powerful and interesting method of attack, which should interest the physicist on account of the clearness with which the results may be interpreted. It would be impossible to cite all the important memoirs that have appeared on this subject, but it would be equally impossible to omit the names of Heaviside, Picard, Poincaré, Boussinesq, Goursat, Brillouin, and Hadamard.

The first appearance of the so-called telegraphist's equation is in a paper by Kirchhoff,* which is remarkable in that it

\footnotetext{
* Kirchhoff, "Ueber die Bewegung der Elektricität in Drähten," Pogg. Ann., vol. 100 (1857); Ges. Abh., p. 131.
} 
appeared before Maxwell's theory of electricity was invented. Twenty years later the equation was first given on the basis of that theory by Heaviside, in a very remarkable paper,* which, like Kirchhoff's, remained unnoticed. Both Kirchhoff and Heaviside used the method of Fourier's series of what are now called normal functions. The treatment of the infinite line began with Poincaré, $\uparrow$ who employed the Fourier integral, Boussinesq, $\ddagger$ who gave a new modification of the method used by Poisson for the wave equation, and Picard, $\$$ who used the method of Riemann.

All these writers were interested in the propagation of the waves as determined by the initial data in the whole line, that is, in the so-called Cauchy problem. Later appeared papers by Brillouin, || in which propagation in three dimensions, and the effect of sources of waves was discussed. But in none of these papers was the problem touched which is often of great importance to the experimental physicist, namely the effect upon the waves of the terminal apparatus, composed of any arrangement of coils, condensers, and resistances, which are connected to one or both ends of a line of finite length, and to which is applied at some point an electromotive force which varies according to a given function of the time. It is true that the influence of the terminal apparatus was completely discussed by Heaviside $T$ in a very powerful paper written in 1882, but published first in 1892, and in a series of others, well characterized by M. Brillouin, as "une prodigieuse série de mémoires à la fois condensés et touffus," in which the method is throughout that of development in series of normal functions. It is for this reason that Heaviside remarks: "it is an enormous and endless subject, admitting of infinite development." It is believed that for this reason anything that will throw light upon or simplify the matter will be of interest.

* Heaviside, "On the extra current," Phil. Mag., Aug., 1876; Electrical Papers, vol. I, p. 53.

$\dagger$ Poincaré, "Sur la propagation de l'électricité," Comptes Rendus, vol. 117 (1893), p. 1027.

$¥$ Boussinesq, "Intégration de l'équation du son pour un fluide indéfini," Comptes Rendus, vol. 118 (1894), p. 162.

\& Picard, "Sur l'équation aux dérivées partielles qui se rencontre dans la théorie de la propagation de l'électricité," Comptes Rendus, vol. 118 (1894), p. 16.

\| Brillouin, "Propagation dans les milieux conducteurs," Comptes Rendus, vol. 136 (1903), p. 667, 16 mars; p. 746, 23 mars.

T. Heaviside, "Contributions to the theory of the propagation of current in wires," Electrical Papers, vol. 1, p. 141 . "On the self-induction of wires, Phil. Mag., 1886-7; Papers, vol. 2, p. 168. 
If $I$ denote the current, $V$ the potential at a point at a distance $z$ from the origin at the time $t$, the equations of propagation are

$$
L \frac{\partial I}{\partial t}+R I=-\frac{\partial V}{\partial z}, \quad K \frac{\partial V}{\partial t}+S V=-\frac{\partial I}{\partial z},
$$

where $L$ is the self-inductance, $K$ the capacity, $R$ the resistance, and $S$ the leakage conductance of the line, all referred to unit of length. If we change the units of length and potential, and remove an exponential factor, putting

$$
\begin{array}{lll}
I=e^{-q t} u, & V=-\sqrt{\frac{L}{K} e^{-q t} v,} & 2 q=\frac{R}{L}+\frac{S}{K}, \\
x=\sqrt{K L \cdot z}, & y=t, & 2 p=\frac{R}{L}-\frac{S}{K},
\end{array}
$$

we reduce to the simple form

$$
\frac{\partial u}{\partial x}=\frac{\partial v}{\partial y}-p v, \quad \frac{\partial v}{\partial x}=\frac{\partial u}{\partial y}+p u,
$$

from which we obtain at once

$$
\frac{\partial^{2} u}{\partial x^{2}}-\frac{\partial^{2} u}{\partial y^{2}}+p^{2} u=0,
$$

which equation is also satisfied by $v$. This is the telegraphist's equation which by the substitution $p x=\xi+\eta, p y=\xi-\eta$ is reduced to the normal form

$$
\frac{\partial^{2} u}{\partial \xi \partial y}+u=0 .
$$

When $p=0$ we have the simple equation for the motion of a stretched string

$$
\frac{\partial^{2} u}{\partial x^{2}}-\frac{\partial^{2} u}{\partial y^{2}}=0 .
$$

This, called by Heaviside the distortionless case, was that treated by Kirchhoff. The researches of Picard, Goursat, and Hadamard have been on the more general equation

$$
\frac{\partial^{2} u}{\partial x \partial y}+a \frac{\partial u}{\partial x}+b \frac{\partial u}{\partial y}+c u=0,
$$

to which most of the results here reached will apply. 
The ordinary, or Cauchy problem, consists in determining the solution by giving the values of $u$ and $\partial u / \partial y$ for the whole line $y=0$. It has been shown by Darboux, Goursat, and Picard, that instead we may give the values of $u$ alone, not giving the normal derivative, along two lines meeting at an angle.* It has been remarked by Hadamard $\dagger$ this is not the problem that interests the physicist, but that, as in the present case, we have Cauchy's data for only a portion of the line $y=0$, while at the end $x=0$ we have a different condition, not being able to give both Cauchy data, but only one, namely the values of $u$. This he terms the mixed problem.

We are here concerned with a line infinite in one direction $\ddagger$ which ends at the point $x=0$, but instead of giving either $u$ or its normal derivative along the line $x=0, y \geqq 0$, we give a relation between them in the form of a linear differential equation in $y$. It may be easily shown that if we have any combination of coils, with self or mutual inductance and resistance, with condensers; connected to the end of the line, with an electromotive force applied somewhere in the combination, the effect will be to make

$$
\begin{aligned}
& a_{0} u+a_{1} \frac{\partial u}{\partial y}+a_{2} \frac{\partial^{2} u}{\partial y^{2}}+\cdots+a_{m} \frac{\partial^{n} u}{\partial y^{m}} \\
& +b_{0} v+b_{1} \frac{\partial v}{\partial y}+\cdots+b_{n} \frac{\partial^{n} v}{\partial y^{n}}=\psi(y), \quad(x=0, y \geqq 0),
\end{aligned}
$$

from which, eliminating $v$ by the first of equations (3) and an equation (18) or (33), deduced below, we get a differential relation between $u$ and the normal derivative $\partial u / \partial x$. Speaking mechanically, the usual end conditions have been $u=0$ for a string fixed at the end, or $\partial u / \partial x=0$ or $\partial u / \partial x-h u=0$ which have been used for other problems. If the string is fastened, however, to a movable point on an apparatus possessed of elasticity, inertia, and dissipation, the condition will be as stated in (8). The writer was led to consider this condition in 1893, from electrical experiments that he was then making, but on account of lack of mathematical knowledge, the question was laid aside. It appears that the method used by Hadamard leads without difficulty to the result.

* See also Mason, New Haven Mathematical Colloquium.

† Hadamard, "Sur un probleme mixte aux dérivées partielles," Bull. de la Soc. Math. de France, vol. 31 (1903), p. 208.

$¥$ If the second end is at a finite distance, the reasoning here given may be applied to it. 
It seems intuitively evident to the physicist that in the present case neither $u$ nor the normal derivative can be given for $x=0$, but that both are completely determined by the condition (8). The calculation shows this to be the case. For simplicity let us first consider the case of no distortion, $p=0$. We have then, if we give the initial distribution of current and potential

$$
\frac{\partial u}{\partial x}=\frac{\partial v}{\partial y}, \quad \frac{\partial u}{\partial y}=\frac{\partial v}{\partial x}, \quad \frac{\partial^{2} u}{\partial x^{2}}-\frac{\partial^{2} u}{\partial y^{2}}=0,
$$

(10) $u=f(x), \quad v=g(x), \quad \frac{\partial u}{\partial y}=g^{\prime}(x), \quad y=0, \quad x \geqq 0$.

Let $P$ be any point with coordinates $\xi, \eta$ from which two characteristics meet the axes in $A, B$, and from $A$ draw the

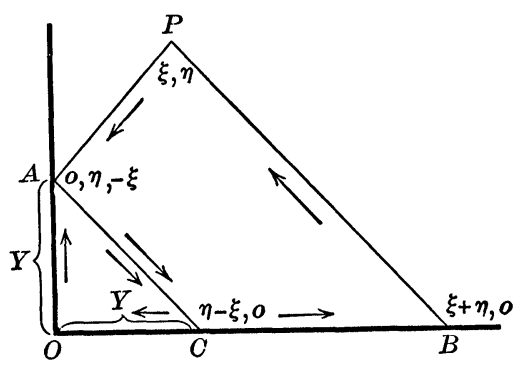

characteristic meeting the $x$-axis in. $C$. The fundamental equation of Riemann's method is

$$
\int G\left\{\frac{\partial u}{\partial x} d y+\frac{\partial u}{\partial y} d x\right\}-u\left\{\frac{\partial G}{\partial x} d y+\frac{\partial G}{\partial y} d x\right\}=0
$$

for a closed contour, where $u$ and $G$ are solutions of the given equation and its adjoint respectively (here the same equation). In the present simple case the function $G$ is constant and equal to unity, so that we have

$$
\int\left\{\frac{\partial u}{\partial x} d y+\frac{\partial u}{\partial y} d x\right\}=0
$$

Applying (12) to the closed quadrilateral $P A C B P$, we have 
1912.] DIFFERENTIAL EQUATION OF TELEgRAPHY.

$$
\begin{gathered}
P A, \quad d x=d y, \quad \int_{P}^{A} d u=u_{A}-u_{P}, \\
B P \\
A C\}, \quad d x=-d y, \quad-\int_{B}^{P} d u=u_{B}-u_{P}, \\
-\int_{A}^{C} d u=u_{A}^{-}-u_{C}, \\
C B, \quad d y=0, \quad \int_{C}^{B} \frac{\partial u}{d y} d x=\int_{C}^{B} \frac{\partial v}{\partial x} d x=v_{B}-v_{C} .
\end{gathered}
$$

Adding these results

$$
2 u_{P}=2 u_{A}+u_{B}-u_{C}+v_{B}-v_{C}
$$

Accordingly it is necessary to determine only the value of $u_{A}$, when we have the result as in Hadamard's case.

If we apply the formula (12) to the triangle $A O C$, we have

$$
0=\int_{o}^{A} \frac{\partial u}{\partial x} d y+u_{A}-u_{C}+v_{o}-v_{C}
$$

so that

$$
u_{A}=u_{C}+v_{C}-v_{o}-\int_{o}^{Y}\left(\frac{\partial u}{\partial x}\right)_{x=0} d y .
$$

Inserting in (14), we have

(17) $u_{P}=\frac{1}{2}\left(u_{B}+u_{C}\right)+\frac{1}{2}\left(v_{B}+v_{C}\right)-v_{O}-\int_{0}^{Y}\left(\frac{\partial u}{\partial x}\right)_{x=0} d y$,

so that the problem is merely shifted to determining the normal derivative $\partial u / \partial x$ along $x=0$. Differentiating (16) by $Y$,

$$
\frac{\partial u(Y)}{\partial Y}=f^{\prime}(Y)+g^{\prime}(Y)-\left(\frac{\partial u}{\partial x}\right)_{x=0}
$$

Differentiating the equation (8) and substituting for $\partial v / \partial y$ its value

$$
\frac{\partial u}{\partial x}=f^{\prime}(y)+g^{\prime}(y)-\frac{\partial u}{\partial y},
$$


we have the linear differential equation

$$
\begin{aligned}
& \left(a_{0}-b_{0}\right) \frac{\partial u}{\partial y}+\left(a_{1}-b_{1}\right) \frac{\partial^{2} u}{\partial y^{2}} \\
& +\cdots=\psi^{\prime}(y)-b_{0}\left(f^{\prime}(y)+g^{\prime}(y)\right)-b_{1}\left(f^{\prime \prime}(y)+g^{\prime \prime}(y)\right)+\cdots .
\end{aligned}
$$

Solving this with the proper initial conditions given by the state of the line and the terminal apparatus at the start completely determines $u_{A}$, so that (14) gives the desired solution. This simple result could have been obtained in a perfectly elementary manner from the solution

$$
u=F_{1}(x+y)+F_{2}(x-y)
$$

or by the method of the Fourier integral.

As a very simple example, let us consider the case of a line initially free from current and potential to which a constant electromotive force $E$ is suddenly applied through a coil of inductance $L_{0}$ and resistance $R_{0}$. Then we have

$$
x=0, \quad L_{0} \frac{\partial I}{\partial t}+R_{0} I+V=E,
$$

or

$$
L_{0} \frac{\partial u}{\partial y}+\left(R_{0}-L_{0} q\right) u-\sqrt{\frac{L}{K}} v=E e^{q y}
$$

and the solution is

$$
\begin{aligned}
& I=0, \quad x>t / \sqrt{K L} ; \\
& I=\frac{E}{R_{0}+\sqrt{L / K}}\left\{1-e^{-\left(R_{0}+\sqrt{L / K}\right)(t-x \sqrt{K L}) / L_{0}}\right\} \quad(0 \leqq x \leqq t / \sqrt{K L}) .
\end{aligned}
$$

We thus see the discontinuity at the wave front, and we find that the line reacts on the terminal system like an extra resistance equal to the inductance of the length of line traversed by the wave in unit of time.

Let us now pass to the case of an actual line $p \neq 0$.

$$
\begin{gathered}
\frac{\partial^{2} u}{\partial x^{2}}-\frac{\partial^{2} u}{\partial y^{2}}+p^{2} u=0, \\
u=f(x), \quad \frac{\partial u}{\partial y}=h(x), \quad y=0, \quad x \geqq 0 .
\end{gathered}
$$


The so-called Riemann function $G$ is not now constant, but is such a solution as is equal to the constant unity along the characteristics $P A, P B$, and accordingly depends not only on the coordinates $x, y$, but on $\xi, \eta$, the coordinates of $P$. We will therefore write $G(x, y, \xi, \eta)$. If we should integrate our formula (11) around $P A C B P$ we should now obtain the integral along the line $A C$, involving values of $u$ which are unknown. To avoid this we integrate along the quadrilateral $P A O B P$, obtaining

$$
\begin{gathered}
P A, \quad \int_{P}^{A} G d u-u d G=(u G)_{A}-(u G)_{P}=u_{A}-u_{P}, \\
B P, \quad \int_{B}^{P} u d G-G d u=(u G)_{B}-(u G)_{P}=u_{B}-u_{P}, \\
A O, \quad \int_{A}^{O}\left(G \frac{\partial u}{\partial x}-u \frac{\partial G}{\partial x}\right) d y, \\
O B, \quad \int_{o}^{B}\left(G \frac{\partial u}{\partial y}-u \frac{\partial G}{\partial y}\right) d x=\int_{o}^{B}\left(G h(x)-f(x) \frac{\partial G}{\partial y}\right) d x, \\
2 u_{P}=u_{A}+u_{B}+\int_{o}^{B}\left(G h(x)-f(x) \frac{\partial G}{\partial y}\right) d x \\
\quad+\int_{o}^{A}\left(u \frac{\partial G}{\partial x}-G \frac{\partial u}{\partial x}\right) d y .
\end{gathered}
$$

As before, we have to obtain the values of $u, \partial u / \partial x$ along $O A$. Proceeding as before, we apply our formula to the triangle $O A C$, but as we wish the Riemann function to be constant along $A C$ we do not use the same $G$ as before, but consider $P$ moved to $A$, so that we put $G_{1}=G(x, y ; 0, Y)$. We thus obtain

$$
\begin{aligned}
u_{A}=u_{C}+\int_{o}^{C}\left(G_{1} h(x)-f(x)\right. & \left.\frac{\partial G_{1}}{\partial y}\right) d x \\
& -\int_{o}^{A}\left(G_{1} \frac{\partial u}{\partial x}-u \frac{\partial G_{1}}{\partial x}\right) d y .
\end{aligned}
$$

We have now to put, according to Picard,

$$
G(x, y ; \xi, \eta)=j\left\{p^{2}\left[(y-\eta)^{2}-(x-\xi)^{2}\right]\right\},
$$

where $j(x)=J_{0}(\sqrt{-x}), J_{0}$ representing the Bessel function of 
order zero. We easily find the values

$$
\begin{aligned}
& \frac{\partial G}{\partial x}=2 p^{2}(\xi-x) j^{\prime}\left\{p^{2}\left[(y-\eta)^{2}-(x-\xi)^{2}\right]\right\}, \\
& \frac{\partial G}{\partial y}=2 p^{2}(y-\eta) j^{\prime}\left\{p^{2}\left[(y-\eta)^{2}-(x-\xi)^{2}\right]\right\},
\end{aligned}
$$

(28) $O B, G(x, 0 ; \xi, \eta)=j\left\{p^{2}\left[\eta^{2}-(x-\xi)^{2}\right]\right\}$,

$$
\begin{aligned}
\frac{\partial G}{\partial y} & =-2 p^{2} \eta j^{\prime}\left\{p^{2}\left[\eta^{2}-(x-\xi)^{2}\right]\right\}, \\
O C, G_{1}(x, 0 ; 0, Y) & \left.=j\left\{p^{2}\left[Y^{2}-x^{2}\right)\right]\right\}, \\
\frac{\partial G_{1}}{\partial y}= & -2 p^{2} Y j^{\prime}\left\{p^{2}\left[Y^{2}-x^{2}\right]\right\}, \\
O A, \quad G(0, y ; \xi, \eta) & =j\left\{p^{2}\left[(y-\eta)^{2}-\xi^{2}\right]\right\}, \\
\frac{\partial G}{\partial x} & =2 p^{2} \xi j^{\prime}\left\{p^{2}\left[(y-\eta)^{2}-\xi^{2}\right]\right\}, \\
G_{1}(0, y ; 0, Y) & =j\left\{p^{2}\left[(y-Y)^{2}\right]\right\}, \quad \frac{\partial G_{1}}{\partial x}=0 .
\end{aligned}
$$

Inserting these in (26)

$$
\text { (29) } \begin{aligned}
u_{A}=u_{C}+\int_{o}^{Y} & \left(h(x) j\left\{p^{2}\left[Y^{2}-x^{2}\right]\right\}+2 p^{2} f(x) Y j^{\prime}\left\{p^{2}\left[Y^{2}-x^{2}\right]\right\}\right) d x \\
& -\int_{o}^{A} j\left\{p^{2}\left[(y-Y)^{2}\right\} \frac{\partial u}{\partial x} d y .\right.
\end{aligned}
$$

All the functions in the first integrand being known, the integral is a known function of its limit $Y$, so that (29) is an integral equation between $u$ and $\partial u / \partial x$ of the form

$$
u(y)=\varphi(y)-\int_{0}^{n} K(x, y) F^{\prime}(x) d x,
$$

where the kernel is symmetrical,

(31) $K(x, y)=j\left\{p^{2}[x-y]^{2}\right\}, \quad K(y, y)=1, \quad F(y)=\left(\frac{\partial u}{\partial x}\right)_{x=0}$, reducing to (16) when $p=0$. 
Differentiating successively by $y$, we obtain

$$
\begin{aligned}
& \frac{\partial u}{\partial y}=\varphi^{\prime}(y)-F(y)-\int_{0}^{y} \frac{\partial K}{\partial y} F(x) d x, \\
& \frac{\partial^{2} u}{\partial y^{2}}=\varphi^{\prime \prime}(y)-F^{\prime}(y)-\left(\frac{\partial K}{\partial y}\right)_{x=y} F(y)-\int_{0}^{y} \frac{\partial^{2} K}{\partial y^{2}} F(x) d x,
\end{aligned}
$$

From the differential equation (3) by integration we have

$$
\frac{\partial v}{\partial y}-p v=F(y), \quad v=\int_{9}^{y} e^{p(y-x)} F(x) d x+v_{0},
$$

from which we may obtain the derivatives of $v$ linearly in terms of those of $F$ and an integral. Substituting all derivatives in the boundary condition (8) we have

$$
A_{0} F(y)+A_{1} F^{\prime}(y)+A_{2} F^{\prime \prime}(y)+\cdots+\int_{0}^{y} \Phi(x, y) F(x) d x=\Psi(y),
$$

which is a linear integro-differential equation of Volterra's form.* Solving this for $F(y)$ and inserting in (29) we obtain the desired solution from (25).

We thus see that the question of the reflection of waves at any terminal apparatus is contained in the values of $u$ and $\partial u / \partial x$ along the line $O A$.

After the preceding paper was completed, I succeeded in seeing Goursat's paper in the Annales de l'Universite de Toulouse, volume 6 (1904), page 117, and found that he had solved the case of $u$ given along two lines making an acute angle, by means of a Volterra integral equation, which is a particular case of the above solution. $\dagger$

Clark University, Worcester, July 5, 1911.

* Rendiconti dell' Acc. dei Lincei, 1909, 1910.

$\dagger$ An abstract of the above paper was presented in the Comptes Rendus, Aug. 28, 1911. 